\title{
Los Programas Universitarios de Mayores y su contribución al aprendizaje a lo largo de la vida
}

\section{The University Programmes for Senior Citizens and their contribution to lifelong learning}

\author{
Ana FERNÁNDEZ-GARCÍA, José Luis GARCÍA LLAMAS y \\ Gloria PÉREZ SERRANO \\ Universidad Nacional de Educación a Distancia
}

Recibido: Mayo 2013

Aceptado: Junio 2013

\section{Resumen}

El estudio ${ }^{1}$ aborda las características esenciales de los Programas Universitarios para Mayores (PUM). Apuesta por la educación a lo largo de la vida mediante la integración de los mayores en la universidad. Parte de la base de que se aprende durante todo el ciclo vital, al no existir ninguna edad, ni espacio específico, para la educación. El aprendizaje se construye desde los conocimientos, capacidades, habilidades y actitudes que se enriquecen continuamente. Es necesario garantizar que los mayores envejezcan de una manera saludable. Se aboga por el envejecimiento activo, herramienta eficaz contra el aislamiento y la exclusión social.

El objetivo consiste en conocer los aspectos del Programa de formación UNED Senior que contribuyen al aprendizaje a lo largo de la vida y mayor satisfacción producen en los implicados.

Los PUM son vías para la promoción del envejecimiento junto a otras actividades dirigidas a potenciar un ocio creativo, acceder a la cultura, facilitar la formación en temas de interés y crear medios de participación en todas las esferas de la sociedad.

En esta investigación se ha recurrido a una metodología mixta. Su riqueza combina los métodos cuantitativos y cualitativos permitiéndonos obtener información y contraste desde diferentes perspectivas. La finalidad es verificar los resultados de la investigación.

La muestra está constituida por 639 alumnos, 57 profesores y 15 coordinadores.

Los resultados indican que el PUM de la UNED Senior es altamente satisfactorio para los implicados, por la adecuación entre intereses y expectativas. Fruto de la presente investigación son las conclusiones y propuestas de mejora.

\footnotetext{
${ }^{1}$ Este artículo presenta los resultados de una investigación AECID. $\mathrm{N}^{\mathrm{o}}$ de referencia (AP/036665/11) 
Palabras clave: investigación; aprendizaje; satisfacción; programa de formación; universidad; vejez.

\begin{abstract}
This study deals with University Programmes for senior citizens (PUM). These programmes are committed to lifelong learning and aim to integrate senior citizens to University life. Bearing in mind that learning takes place throughout life, there is no age limit, no specific space for education. Learning involves constructing knowledge and takes advantages of skills, abilities and aptitudes to benefit from one another. We believe that healthy and active ageing are effective tools against isolation and social exclusion. The aim of this study is to learn which aspects of the Spanish Open University (UNED) senior training programme contribute to lifelong learning and major satisfaction among the participants. These programmes also carry out leisure activities, allowing senior citizens to access culture, facilitate training on topics of their interest and foster participation in all spheres of society. This research study uses a mixed methodology (quantitative and qualitative research methods) in order to gather information from different perspectives. The sample consisted of 639 students, 57 teachers and 15 coordinators. The results indicate that the PUM in the UNED is highly satisfactory since it meets the participants' expectations and interests. Finally, we will draw some conclusions and recommendations
\end{abstract}

Keywords: research; learning; satisfaction; training program; university; old age.

El aumento de la esperanza de vida en la sociedad occidental, junto con los cambios producidos en el ámbito laboral han ocasionado, desde finales del siglo XX, la aparición de un gran sector de población laboralmente inactivo, que se encuentra en situación de jubilación. Cabe destacar que este colectivo, en general, goza de buena salud y se encuentran en pleno uso de sus facultades para decidir y aprovechar su tiempo.

Esta nueva situación propicia que las personas mayores experimenten fuertes cambios en su forma de vida. Los cambios son positivos en este colectivo cuando gana en autonomía e independencia, es decir, disfrutando de más tiempo de ocio, participando activamente en asociaciones, organizaciones, en la Universidad..., que contribuyen al desarrollo y crecimiento personal en su nueva vida.

No obstante, existe un sector minoritario de población mayor que envejece con grandes dificultades, tanto físicas como intelectuales. Vivir más años no significa, en esencia, una mejor calidad de vida, puesto que, "las personas mayores no se encuentran muchas veces con los recursos personales idóneos para adaptarse a situaciones y nuevas maneras de ver la realidad y de actuar en ella" (Bermejo, 2010).

Las constantes transformaciones que experimenta la sociedad actual, ocasionan drásticos y fuertes cambios en todos los ámbitos, tanto económicos, culturales, sociopolíticos, como tecnológicos...

Desde diversos sectores sociales se reclama la creación de modalidades y propuestas formativas que les aporten nuevas oportunidades, pero que no constituyan un mero pasatiempo sino que les permita seguir activos intelectualmente; así como 
frecuentar ambientes distintos $\mathrm{y}$ fomentar las relaciones interpersonales $\mathrm{e}$ intergeneracionales con la finalidad de contribuir a su plena integración social.

Sin duda, es necesario garantizar que los mayores envejezcan de una manera saludable y activa. Por todo ello, abogamos por el envejecimiento activo, una herramienta eficaz para luchar contra el aislamiento y la exclusión social, haciendo partícipes a los protagonistas en la vida económica, política, social, educativa y cívica; a ello contribuyen los Programas Universitarios para Personas Mayores (PUM).

Los objetivos que a continuación se presentan tienen por finalidad guiar el proceso de investigación.

El objetivo general consiste en conocer los aspectos del Programa de formación UNED Senior que contribuyen al aprendizaje a lo largo de la vida y que más satisfacen a los implicados (alumnos, profesores y coordinadores).

Para su consecución, se plantean los siguientes objetivos específicos:

- Definir el perfil de los alumnos, profesores y coordinadores que participan en el Programa.

- Examinar los motivos que impulsan a los adultos mayores a matricularse.

- Estimar el grado de satisfacción de los alumnos, profesores y coordinadores de la UNED Senior con el Programa.

- Detectar el grado de satisfacción de los alumnos con el profesorado.

- Analizar si los centros poseen recursos suficientes para desarrollar el Programa con calidad.

- Elaborar propuestas de mejora.

\section{Aproximación al concepto de envejecimiento activo}

La Organización Mundial de la Salud (OMS, 2002) define el envejecimiento activo como "el proceso por el que se optimizan las oportunidades de bienestar físico, social y mental durante toda la vida, con el objetivo de ampliar la esperanza de vida saludable, la productividad y la calidad de vida en la vejez".

Tomando como referencia a Bermejo (2010), desglosamos el término envejecimiento activo, señalando algunos de sus elementos esenciales:

1. Expresa la idea de la participación continua de las personas en las cuestiones sociales, económicas, culturales, espirituales y cívicas, sin centrarse sólo en la capacidad para estar física y laboralmente activo.

2. Requiere que la sociedad garantice que los ciudadanos mayores puedan continuar informados, a la vez que vela porque sean reconocidos todos sus derechos.

3. Asume un enfoque intergeneracional al reconocer la importancia de las relaciones y de la ayuda que se ofrecen entre sí los miembros de una y otra generación.

4. Requiere un enfoque integral, pues ofrece una visión más completa y global de las personas y de su proceso de envejecimiento. 
5. Desafía la perspectiva tradicional de que el aprendizaje es cuestión de niños y jóvenes, el trabajo es cuestión de adultos y la jubilación es cuestión de la vejez. Sin embargo promueve el desarrollo de la persona a lo largo de todo el ciclo vital.

Como queda reflejado en el Libro Blanco (IMSERSO, 2011) el envejecimiento activo constituye, en definitiva, un aspecto fundamental para combatir y demorar situaciones de dependencia tanto físicas, como psíquicas, sociales, educacionales y funcionales.

Es significativo destacar que los PUM desempeñan un importante papel en su promoción y desarrollo. Esta reflexión se abordó en la Jornada Científica denominada "La Formación Permanente en la Estrategia Europea", celebrada en Madrid en junio de 2007, donde se destacó este hecho al afirmar que los programas de formación universitaria son un medio de intervención eficaz para prevenir situaciones de dependencia y, por ende, constituyen un elemento clave en la potenciación de un envejecimiento activo y saludable, cuya finalidad es mejorar la calidad de vida de los adultos mayores.

La preocupación por estas cuestiones ha estado presente en los últimos tiempos a nivel nacional e internacional. Es importante tener en cuenta que, el envejecimiento activo, necesita el apoyo de una amplia gama de políticas en todos los niveles de gobierno. La organización de Conferencias y Asambleas ha impulsado su desarrollo y la expansión del aprendizaje a lo largo de la vida, elemento esencial para su consecución. Se destacan dos Asambleas Mundiales del Envejecimiento celebradas en Viena (1982) y en Madrid (2002), respectivamente.

En la I Asamblea Mundial del Envejecimiento: Plan de Viena (1982) se reconoce por primera vez, la dimensión educativa en la vejez y la importancia del desarrollo de la educación con el objetivo de obtener una mejor calidad de vida. Se enumeran tres modalidades principales en la acción educativa en la tercera edad:

1. Actuación basada en medidas preventivas para la tercera edad

2. Mejorar la situación de las personas ya jubiladas desde la acción cultural y social

3. Actuación encaminada a educar a la sociedad en relación con los senescentes.

La II Asamblea Mundial del Envejecimiento, celebrada en Madrid (2002) con el lema "Una sociedad para todas las edades" tuvo como propósito guiar la formulación de políticas hacia el objetivo de lograr durante toda la vida y en toda la sociedad el ajuste social, cultural y económico a un mundo que envejece. En la Orientación Prioritaria I (cuestión 4) de la citada Asamblea se señala la importancia de la formación y educación en la vejez.

Asimismo en la Conferencia Ministerial sobre Envejecimiento celebrada en Viena en el año 2012, bajo el lema "Garantizar una sociedad para todas las edades: la promoción de la calidad de vida y el envejecimiento activo", se reitera la importancia de la promoción del aprendizaje a lo largo de la vida para desarrollar el potencial de las 
personas mayores. Las áreas temáticas tratadas dieron lugar a la Declaración Ministerial de Viena 2012 y hacen referencia a:

- Promover la participación, la no discriminación y la inclusión social de las personas mayores.

- Creación de un entorno saludable para la salud, la independencia y el envejecimiento digno.

- Impulso del diálogo y la solidaridad entre generaciones: responsabilidad compartida.

- Promoción de la extensión de la vida laboral y mantenimiento de la empleabilidad.

Gracias a estos eventos y acciones han ido surgiendo muchos programas socioeducativos (Jiménez y Palmero, 2012), entre los cuales destacan los PUM, que hacen posible el deseo de aprender a lo largo de la vida. Proporcionan a los adultos mayores una actividad socialmente relevante que les permita adaptarse a las exigencias de la sociedad científica y tecnológica actual.

\section{Importancia del Aprendizaje a lo largo de la vida}

El Informe Delors denominado "La educación encierra un tesoro" (1996), defiende la Educación Permanente, sin límites de espacio o edad, capaz de dar respuesta a una profunda necesidad de cultura y de actualización de unos adultos que ahora disfrutan de una mayor longevidad a la que desean sacar el máximo provecho.

La educación permanente (cuyos orígenes se remontan a Comenius en el siglo XVI, aunque su verdadero precursor ha sido Grundtvig en el siglo XIX) sustenta la idea de la educación a lo largo de la vida (surgida a partir de la década de los 70 del siglo XX). Se suele utilizar para hacer referencia a ámbitos no escolares. Por ello puede aplicarse a los adultos mayores. Su objetivo consiste en mejorar su calidad de vida y su participación.

En el presente artículo se apuesta por la educación a lo largo de la vida, mediante la integración de los mayores en la Universidad. Se parte de la base de que se puede aprender durante todo el ciclo vital, al no existir ninguna edad ni espacios específicos para la educación. El periodo de aprendizaje de una persona debe abarcar toda la vida. Se alimenta mediante la construcción de conocimientos, capacidades, habilidades y actitudes que se enriquecen continuamente. Es necesario generar una cultura de calidad que, no se oriente sólo a la reflexión y al desarrollo de habilidades técnicas sino que contemple también la dimensión emocional.

Abrir las puertas de la Universidad a personas de todas las edades, es un desafío que, además ofrece la posibilidad de dar respuesta a una demanda creciente de formación de este colectivo social. Es importante destacar que la educación a lo largo de la vida no es un ideal sino una realidad que se evidencia cada vez más en el ámbito de la educación. Una pequeña inversión hoy en la educación de los mayores, puede 
significar mañana un ahorro muy importante en la atención a la dependencia y sanidad (Libro Blanco, IMSERSO, 2011).

El aprendizaje a lo largo de la vida contribuye a que todos los ciudadanos tengan la posibilidad y oportunidad de satisfacer sus aspiraciones, elevar el nivel educativo, favorecer la participación en la sociedad y reducir las desigualdades.

Conviene tener presente que la oferta educativa destinada a los mayores debe realizarse con la máxima calidad, procurando una oportunidad de crecimiento y una plena autorrealización en la sociedad. Esta debe ser consciente de que el aprendizaje a lo largo de la vida favorece la calidad de vida de las personas mayores.

\section{Los programas Universitarios de Mayores en España}

En la última década del siglo XX surgen en España los PUM que, con diferentes denominaciones (Universidad de la Experiencia, Aula de la Experiencia, Universidad de Mayores, etc.) tratan de dar respuesta a una educación de carácter universitario para las personas mayores en la misma institución universitaria (AEPUM, 2012).

Según esta asociación, se pueden considerar PUM aquellas ofertas educativas, dirigidas preferentemente a personas mayores de 50 años, en las que, de uno u otro modo, participa la Universidad.

Los PUM nacen motivados por el fenómeno demográfico del rápido envejecimiento poblacional y por las demandas de una participación social y cultural activa. Con su creación, las universidades se convierten en promotoras y difusoras de la cultura entre la comunidad universitaria y la sociedad en su conjunto. Tienen por finalidad la formación integral de la persona en su proceso de educación permanente.

Todo ello es posible gracias al apoyo de diversas instituciones, entre ellas, las Consejerías de Servicios Sociales de distintas Comunidades Autónomas, el Ministerio de Trabajo y Asuntos Sociales (IMSERSO), Fundaciones y Bancos.

Los programas de mayores propician el desarrollo científico, cultural y social, la mejora de la calidad de vida de los implicados, el desarrollo personal y cultural, así como el fomento de la participación en su contexto como dinamizadores sociales. Así lo corrobora el Libro Blanco sobre Envejecimiento Activo (IMSERSO, 2011):

"Los Programas Universitarios para Mayores (PUM) también presentan ventajas para sus alumnos, abren espacios educativos "valiosos" a este grupo poblacional: poseen una gran eficacia simbólica por la presencia de las personas mayores en la Universidad; permiten la convivencia y el encuentro intergeneracional; favorecen el desarrollo del asociacionismo y del intercambio entre alumnos de universidades de otras Comunidades Autónomas y otros países; permiten abrirse a oportunidades en experiencias de aprendizaje más complejas (como las TICs) y participar en investigaciones y en proyectos de carácter europeo. Además es muy 
importante valorar los esfuerzos de (estos) programas por mejorar sus procesos de enseñanza-aprendizaje" (p. 300)

Actualmente la presencia de PUM es muy numerosa, vinculados tanto a universidades públicas como privadas. Cada Universidad diseña su propio Programa, no obstante, según se recoge en el Libro Blanco sobre Envejecimiento Activo (IMSERSO, 2011) pueden distinguirse tres objetivos importantes, compartidos por todos ellos, en cuanto a su metodología:

- Intentan adaptar los contenidos al nivel de formación de los participantes.

- Pretenden tener en cuenta las situaciones específicas del aprendizaje de las personas mayores.

- Buscan que las clases se complementen con otras actividades de carácter sociocultural.

Es significativo destacar que la Declaración Mundial sobre la Educación Superior en el siglo XXI de la UNESCO establece, como una acción prioritaria, que el acceso a los establecimientos de Educación Superior debe abrirse a los educandos adultos, creando oportunidades de aprendizaje de manera flexible, abierta y creativa. Por ello, los programas los diseñan y desarrollan cada universidad, con el fin de adaptar sus propuestas a la evolución que demanden los participantes.

Los PUM, cumplen una gran función social; no sólo benefician a los adultos mayores que asisten a sus aulas, sino también a la propia Universidad que ve proyectada su acción cultural, científica e investigadora en otros sectores de la sociedad que reclaman sus servicios y a los que la Universidad debe abrirse y contribuir de este modo, a universalizar la cultura.

Las Universidades proporcionan a los alumnos mayores una formación de carácter superior que está dando lugar a uno de los proyectos más novedosos que contribuye a cambiar la fisonomía de estas instituciones.

En España, esta acción formativa se ampara en lo establecido por la Ley Orgánica de Universidades (LOU) en su Título Preliminar, art. 1, apartado d), considera como misión de la universidad la difusión del conocimiento y la cultura a través de la extensión universitaria y la formación a lo largo de toda la vida. El Título VI, art. 34.3, señala que las universidades podrán establecer enseñanzas conducentes a la obtención de diplomas y títulos propios, así como enseñanzas de formación a lo largo de la vida. Asimismo, la Ley Orgánica 4/2007, que modifica la anteriormente citada, subraya la importancia del aprendizaje a lo largo de la vida en el desarrollo personal, cultural, científico, técnico y económico. 


\section{La UNED Senior: principales agentes implicados}

La Universidad Nacional de Educación a Distancia (UNED) creó el programa universitario denominado, UNED Senior. Se dirige a la formación de adultos-mayores de 55 o más años. Ofrece la oportunidad de asistir a la Universidad a través de un programa específico. La UNED Senior es un programa formativo que contiene temas de actualidad y estrategias de desarrollo personal dirigido a los adultos mayores con la finalidad de ofrecer una formación de calidad que responda a los intereses, demandas y expectativas de este colectivo. Constituye un espacio universitario propio, donde se intercambian conocimientos y experiencias. Proporciona una formación cercana al lugar de residencia de cada estudiante, gracias a la existencia de Centros Asociados ubicados no sólo en toda la geografía española sino también en el extranjero. Se apoya en las tecnologías de la información y la comunicación.

Los agentes implicados en el Programa de la UNED Senior, son los alumnos, los profesores y los coordinadores que comparten esta experiencia educativa. Cada uno de ellos posee una serie de características y obligaciones que definen y fortalecen la identidad del Programa, a saber:

El alumnado se caracteriza por tener más de 55 años. Acoge a todas aquellas personas que lo soliciten con independencia de la formación académica que posean. También permite e impulsa la matriculación de sujetos con necesidades especiales. Esta formación se dirige a personas que, por diversas razones, no pudieron acceder a la Universidad en su momento, o bien desean volver a las aulas y reencontrarse de nuevo con las actividades académicas universitarias con el fin de enriquecer sus conocimientos.

En cuanto al personal docente se pueden distinguir dos grupos de profesores, los docentes de la Sede Central y los profesores-tutores. Los primeros se encargan de elaborar la guía didáctica, la cual orienta al profesor tutor y al alumno. Los profesorestutores cuentan con gran autonomía e iniciativa en el proceso de enseñanzaaprendizaje. Diseñan los objetivos y seleccionan los contenidos más pertinentes para cada asignatura. Es el docente más cercano, que está en contacto directo con los alumnos. Son los encargados de desarrollar el programa e impartir las clases y asesorar a los alumnos semanalmente adecuando los contenidos y la metodología.

Los coordinadores realizarán su labor en plena relación y colaboración con la dirección de la UNED Senior. La función de los coordinadores consiste en: velar por la calidad del programa educativo, adaptarse a la metodología inherente de la UNED y satisfacer las expectativas de los estudiantes.

La organización funcional se caracteriza por su flexibilidad. Tiene como finalidad velar por la calidad de los diferentes elementos necesarios que contribuyen al buen funcionamiento. Los coordinadores desempeñan un papel muy significativo e imprescindible para el funcionamiento del Programa constituyendo el nexo de enlace entre la universidad, los profesores y los alumnos. 


\section{Metodología}

Para llevar a cabo esta investigación se consideró pertinente recurrir a una metodología mixta: de carácter cuantitativo y cualitativo. La riqueza de la misma radica en que se pueden combinar armónicamente ambas metodologías, permitiéndonos obtener mayor información y contrastarla desde diferentes perspectivas con la finalidad de verificar los resultados de la investigación. El método, como expresa Pérez Serrano (2011, p. 28), "es un elemento esencial en la estructura del trabajo científico. Es imprescindible para la búsqueda de un resultado que no está determinado".

La recogida de datos se llevó a cabo a través de tres cuestionarios elaborados a tal efecto, uno para los alumnos, otro para los profesores y el último dirigido a los coordinadores. Permiten recoger la información proporcionada por los implicados. Asimismo, se realizaron grupos de discusión con los profesores de los diferentes centros que imparten el Programa.

Para realizar los análisis estadísticos se empleó el programa SPSS, versión 17.0. Se han realizado análisis descriptivos y análisis multivariantes (clúster y segmentación). Para el análisis cualitativo (textual y conceptual) se utilizó el programa Atlas.ti 7.0.

La fiabilidad de los instrumentos se calculó mediante el coeficiente Alpha de Cronbach. Los valores obtenidos han sido superiores a 0,80 - mínimo recomendado (Henson, 2001),- en concreto el coeficiente Alpha obtenido ha sido de 0,889 (cuestionario alumnos); de 0,876 (cuestionario profesores) y de 0,892 (cuestionario coordinadores). Estos resultados nos indican un nivel alto de consistencia interna.

La población de referencia es el conjunto de alumnos, profesores y coordinadores que forman parte de los diferentes Centros Asociados repartidos por todo el territorio español y del extranjero. Los datos se recogieron en el Curso Académico 2012-2013. La muestra final se calculó con criterios de representatividad y muestreo estratificado con asignación proporcional. En él, la muestra se distribuye entre los estratos proporcionalmente a los tamaños de éstos. Del total de los 85 docentes, han participado en esta investigación 57, aproximadamente el $67 \%$. Del total de 2180 alumnos, han participado en la investigación 639 , el $29,31 \%$. De un total de 20 coordinadores han participado 15, el 75\%. La muestra es representativa de la población objeto de estudio.

El proceso de recogida de información se llevó a cabo, en primer lugar, a través de la aplicación de los cuestionarios en los diferentes Centros Asociados donde se desarrolla el Programa de formación. No se ha considerado necesaria la identificación personal de los sujetos de la muestra, puesto que los datos que nos interesaba recoger en los cuestionarios están referidos a su situación personal y contextual, sus opiniones y/o valoraciones, etc. Previamente se les informó que los datos obtenidos iban a ser utilizados a efectos de estudios de investigación y no como proceso de control de su propia actividad docente. Ello nos ha permitido una mayor naturalidad en las respuestas. Este proceso se llevó a cabo en los meses de febrero-marzo de 2013.

En segundo lugar, se ha recogido información de los grupos de discusión. Se realizaron cinco grupos entre los profesores de los Centros Asociados de: Madrid, A 
Coruña, Pamplona, Xátiva y Pontevedra, elegidos por albergar el mayor número de alumnos. Cada uno de los grupos estuvo formado por 5-7 profesores de cada centro. Al finalizar cada debate, aproximadamente de una hora de duración cada uno, se ha realizado el reporte de sesión donde se han recogido los aspectos más relevantes obtenidos a través de esta técnica, referidos a los aspectos positivos y las sugerencias de mejora respecto al Programa formativo. Todos ellos ayudaron a alcanzar la finalidad de esta investigación.

En relación a las técnicas de análisis de datos para el análisis cuantitativo se ha llevado a cabo a través del paquete estadístico SPSS, siguiendo diferentes etapas:

Se recurrió al análisis de tipo descriptivo permitiendo establecer, de forma básica, los perfiles de los participantes en sus rasgos personales (sexo, edad, nivel de estudios, etc.). Así mismo se obtuvieron las valoraciones en torno a los diferentes elementos del programa.

Seguidamente se han realizado los análisis de tipo inferencial que cumplen una función complementaria. Mejoran el análisis de los datos y ofrecen una visión global de los mismos. Para este fin, se fija un margen de error de 0,05 ; lo que equivale a un nivel de confianza del 95\%. Los análisis ejecutados han sido los siguientes: contrastes mediante el ANOVA, la prueba "t" de Student, la " $z$ " de Fisher y la prueba de Ji Cuadrado.

Finalmente, con el análisis multivariante, se han contemplado aquellos datos que ponen de relieve diferencias significativas entre los grupos del estudio. Los procedimientos utilizados para el análisis son los siguientes:

El análisis de clúster o conglomerados, conocido también como "reconocimiento de patrones". Su finalidad es la formación de grupos. Es una técnica de análisis exploratorio de datos para resolver problemas de clasificación. Su objetivo consiste en ordenar objetos (variables con nivel de medida ordinal en nuestro estudio) en grupos de forma que el grado de asociación/similitud entre miembros del mismo clúster sea más fuerte que el grado de asociación/similitud entre miembros de diferentes clúster.

El análisis de segmentación permite crear un modelo de clasificación basado en árboles, "árbol de decisiones". Este procedimiento pronostica valores de una variable (criterio) dependiente basada en valores de variables independientes (predictores). Se ha utilizado el método de crecimiento CHAID (CHi-square Automatic Interaction Detection). En cada paso CHAID presenta la interacción más fuerte de la variable independiente con la variable dependiente fundiéndose las que no sean significativas. Este método examina todas las divisiones posibles y muestra la salida mediante un "árbol” clasificatorio de fácil interpretación.

Para el análisis de la información cualitativa procedente de los grupos de discusión se ha utilizado el programa ATLAS.Ti. Con este programa se realizaron análisis textuales y conceptuales. Los grupos de discusión aportaron opiniones y sugerencias de gran valor para detectar las motivaciones, intereses y necesidades de los implicados en el Programa. 


\section{Análisis e interpretación de los resultados}

A continuación se exponen los resultados obtenidos en la investigación. En primer lugar se recogen someramente, las respuestas más importantes obtenidas por los alumnos y coordinadores implicados en el programa formativo de la UNED Senior, para posteriormente, centrarnos en los resultados obtenidos por los profesores tras la aplicación de los respectivos cuestionarios. De la información obtenida con los instrumentos utilizados, se ha realizado una selección de datos acorde con los objetivos de este trabajo.

\section{Alumnos}

La muestra de esta investigación corresponde a alumnos participantes de diferentes Comunidades Autónomas españolas, en los centros de zona (Tortosa, Pontevedra, Plasencia, Madrid, Barbastro, Sant Boi, Xátiva, Pamplona, Cantabria, Garrotxa, A Coruña, Vila real, Madrid Sur, Burgos y Tenerife) y un centro del extranjero (BernaSuiza).

En relación al perfil de los alumnos que participan en el Programa conviene indicar que el 15,5\% tiene más de 70 años. Predominan las mujeres matriculadas en un $59 \%$. El nivel de estudios es muy variado dependiendo del centro; no obstante, cabe destacar que un $34,9 \%$ de los participantes poseen estudios universitarios. Por lo que se refiere a la situación laboral, como era de esperar, predominan los jubilados en un $72 \%$ y se halla en activo un 14,6\%. En cuanto al estado civil, convive en pareja el 68,9\%; solteros el $8,8 \%$; viudos un $15,3 \%$ y divorciados el $4 \%$.

Los motivos por los que se matricularon en el programa son: fundamentalmente, para aprender más, valorado por un $86,3 \%$; seguido de mantenerse activo en un 78,6 $\%$. Conseguir un diploma constituye el aspecto menos valorado, tan solo el 18,6\%. Este último aspecto se confirma en los análisis de clúster y análisis de segmentación al concluir que conseguir un diploma no constituye una prioridad para los alumnos matriculados en el Programa.

El 89,7 \% también opina que les gustan las actividades programadas. El trabajo en grupo es menos valorado, alcanza el $68 \%$.

La opinión de los alumnos sobre el profesorado es altamente positiva. Así lo manifiestan en aspectos tales como: el conocimiento de la materia por parte del profesor valorado por el 95,2 \% de los participantes; seguido por, el profesor explica de forma clara, que alcanza el $94,5 \%$. El profesor utiliza ejemplos y tiene en cuenta nuestras opiniones en un $92,2 \%$.

En cuanto a la dimensión organizativa el aspecto más valorado, el $86,5 \%$, es el funcionamiento del Programa en el Centro Asociado.

Se recogió también información del alumnado sobre si la UNED Senior satisface sus expectativas y si continuaría el curso siguiente. Contestan afirmativamente el 87,2 $\%$. 
El grado de satisfacción es bastante alto alcanzando una media de 8,4 en una escala de 1 a 10. Realizado un análisis de segmentación mediante el procedimiento CHAID, se obtiene que la mayor satisfacción del alumnado se manifiesta en el buen funcionamiento del Programa. Les gustan las actividades que se han programado en sus respectivos Centros Asociados, afirmando que la UNED Senior responde a lo que esperaban.

\section{Coordinadores}

Los coordinadores desempeñan un papel significativo e imprescindible para el desarrollo del Programa. El 53\% son varones y el 47 \% son mujeres. En cuanto a la edad se hallan en la década de los 30-40, tres; coordinadores; en la década de los 50, cinco coordinadores y en la de los 60 o más, siete coordinadores. El nivel de estudios es alto, todos son licenciados y el $46,7 \%$ doctores.

Se ha recogido información sobre si han realizado campañas de motivación. Contestan afirmativamente el $93,4 \%$. Un $28,7 \%$ manifiestan que todavía se conoce poco el programa en su zona. El $80 \%$ de los coordinadores indica que el Centro Asociado dispone de recursos suficientes para impartir el Programa. Las instalaciones son adecuadas para el $93,4 \%$.

Los recursos personales son altamente valorados por el 93,3\%. Todos los coordinadores consideran que el clima existente en el centro favorece el funcionamiento del Programa. Valoran muy positivamente la vinculación de profesores y alumnos con el Centro Asociado.

Un aspecto altamente valorado por los coordinadores es la participación de los mayores en la comunidad que alcanza un 93,3\%. También consideran que el Programa ayuda a los mayores a reflexionar sobre sus posibilidades y la aportación que realizan a la sociedad el $86,7 \%$. La mayoría de los coordinadores, el $86,7 \%$ indican que se tiene en cuenta las opiniones y deseos expresados por los alumnos.

Valoran positivamente las materias que se imparten en el Centro Asociado, el 80\% de los coordinadores. Así mismo, aprecian el esfuerzo del profesorado por adaptarse al colectivo al que se dirigen, teniendo en cuenta las peculiaridades del aprendizaje adulto.

Estiman competentes a los profesores que imparten el programa en un $93 \%$. Valoran, también, su alto nivel de cualificación, la capacidad de preparar el curso y su preocupación por la orientación y seguimiento de los adultos mayores.

Para finalizar conviene resaltar el alto nivel de satisfacción de los coordinadores que alcanza una media de 8,35 en un recorrido de uno a diez. Realizado un análisis de segmentación mediante el procedimiento CHAID, se obtiene que la mayor satisfacción de los coordinadores se genera al constatar que los profesores y alumnos están satisfechos con la organización y el funcionamiento del centro. 


\section{Profesores}

El perfil de los profesores se caracteriza por los siguientes rasgos: son varones $(54,4 \%)$; y, el 75, 4\% menores de 50 años. Por lo que respecta al nivel de estudios el $59,6 \%$ son licenciados y el $21,1 \%$ doctores. En tanto que el $10,5 \%$ son diplomados.

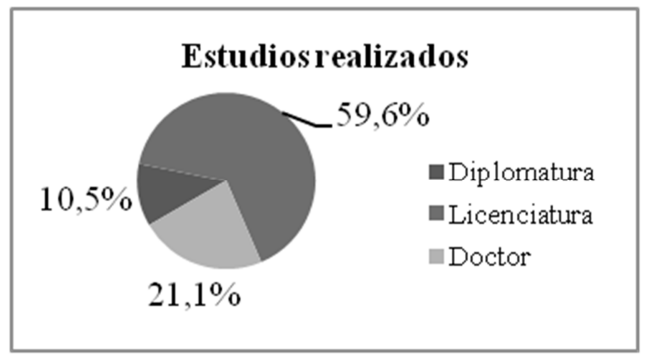

Gráfico 1. Estudios realizados (profesores)

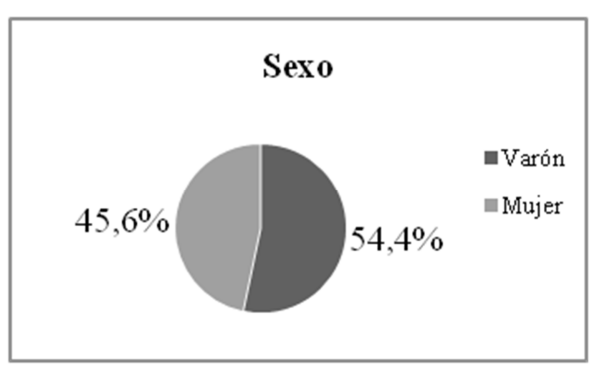

Gráfico 2. Sexo (profesores)

Cabe destacar que su experiencia docente con los adultos mayores no es dilatada. Tan solo el 61,4\% cuenta con 4 años o más de experiencia; no obstante, afirman en un $54,4 \%$ que en otros niveles educativos han desempeñado su función docente a lo largo de 11 o más años.

En cuanto a la planificación que realiza el profesor de adultos mayores se solicitó su opinión sobre los aspectos a los que prestaban una mayor atención. Entre ellos destacan: utilizar métodos que favorecen el aprendizaje adulto el 98,3\% seguida de vincular la teoría-práctica en un $98,3 \%$ y un $71,9 \%$ consideran importante partir de las experiencias y conocimientos previos de los participantes. El análisis de clúster realizado corrobora estos datos, reiterando la importancia de las variables reseñadas anteriormente.

Al programar afirman tener en cuenta la selección, organización y secuenciación de los contenidos, bastante un 54,4\% y mucho un $47,1 \%$. Programan actividades para afianzar los conocimientos el 96,5\%; en cambio no suelen programar actividades para trabajar en equipo un 30,3\%. Sin embargo programan buscando la aplicación en la práctica de lo aprendido un $61,23 \%$.

Los métodos empleados para el aprendizaje adulto suelen ser variados. Las modalidades de trabajo más utilizadas en las clases son los debates que alcanzan un $73,7 \%$ y el trabajo personal independiente un $70,2 \%$. La clase magistral, por el contrario tan solo la utiliza el 56,1\%.

Los recursos y materiales más utilizados por el profesor son los apuntes propios $86,6 \%$ y las fotocopias el $76,7 \%$. Apenas recurren a los blogs y revistas científicas. Aspectos que se corroboran en el análisis de clúster realizado. 


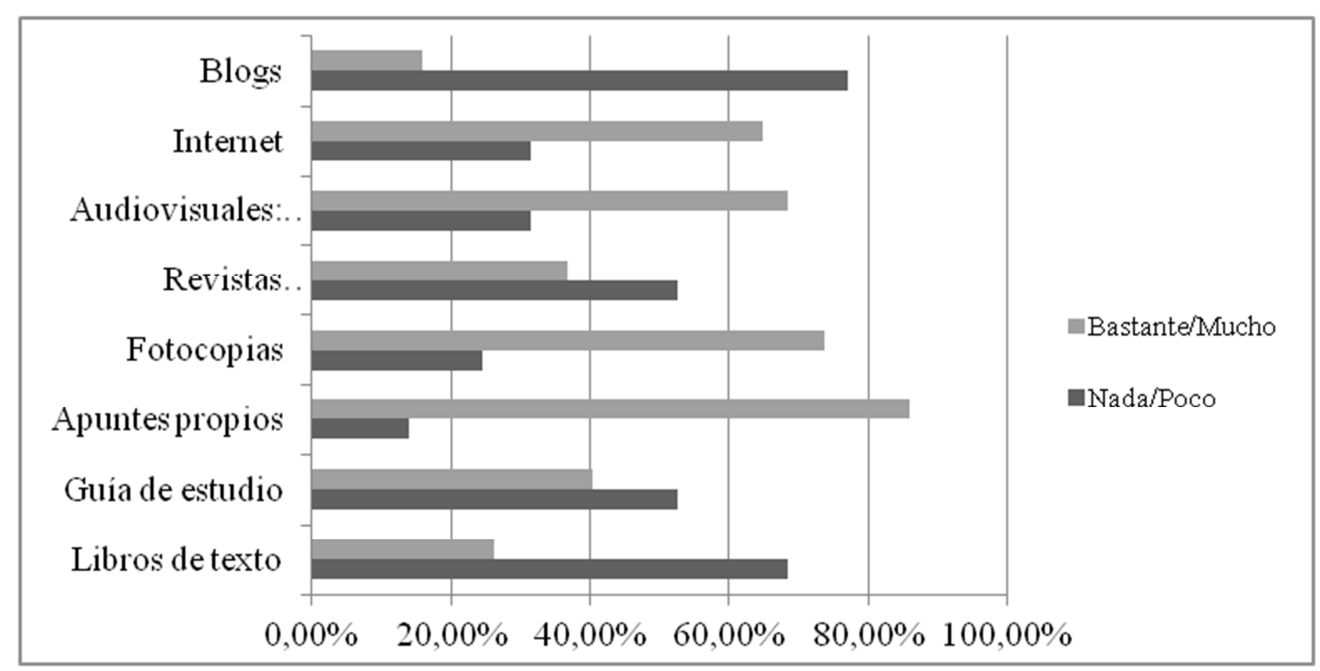

Gráfico 3. Recursos y materiales didácticos

El profesorado en sus clases presenciales presta especial atención por encima del 96\% a los siguientes aspectos: sintetizar los puntos clave; promover la participación e interacción; y destacar los conceptos eje y relacionarlos con otros.

En cuanto a la exposición que realizan a los alumnos, los profesores valoran especialmente, por encima del $90 \%$ el ser asequible al grupo de alumnos; apoyarse en sus comentarios y buscar aplicaciones concretas de lo aprendido. Estas variables resultan de gran importancia en el análisis de clúster realizado puesto que manifiesta la relevancia otorgada por los profesores.

Los profesores que trabajan con personas mayores valoran el 98,3\% tener habilidad para trabajar con este colectivo y manifiestan que es necesario estar motivado para este tipo de enseñanzas.

El profesorado mantiene relaciones de contacto con el centro, además de sus clases, el $77,2 \%$.

La guía didáctica la consideran imprescindible el $68,4 \%$, necesaria el $49,1 \%$ y poco útil el 49,1\%.

Los profesores opinan que las instalaciones del centro asociado son adecuadas para el $80,6 \%$. Los recursos informáticos son suficientes para el $68,4 \%$, en tanto que, en algunos centros asociados son escasos.

Es altamente valorado con el $94,7 \%$ el personal que trabaja en el centro asociado puesto que contribuye a solucionar sus problemas.

El profesorado tiene un nivel de conocimiento muy dispar del Programa UNED Senior. El 80,7\% afirma conocerlo bastante en tanto que el 19,3\% indica que lo conoce poco. 


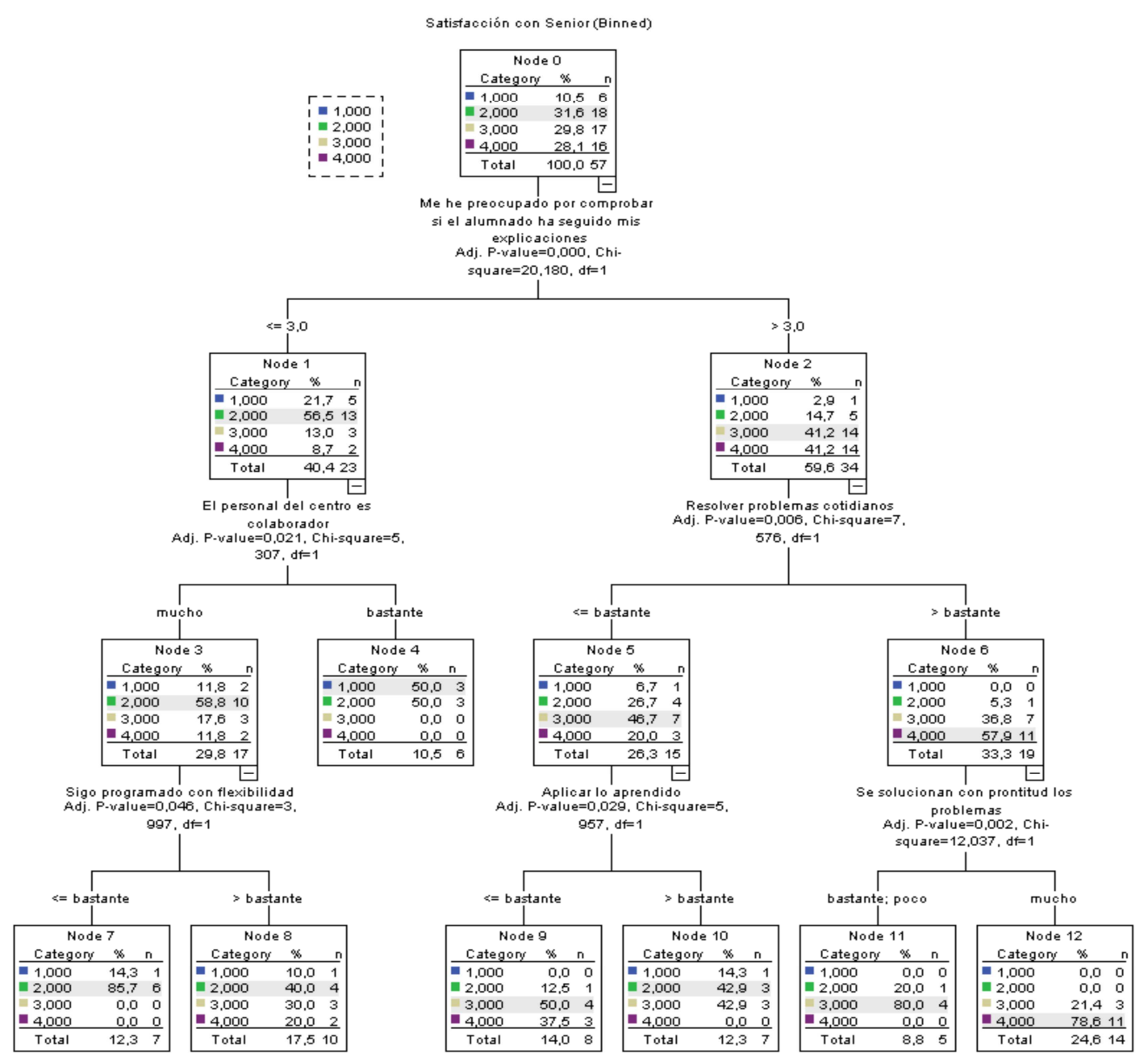

Gráfico 4. Árbol de segmentación

El nivel de satisfacción del profesorado con el Programa alcanza, en un recorrido de 1 a 10 una media de 8,75. Realizado un análisis de segmentación mediante el procedimiento CHAID, se obtiene que la alta satisfacción del profesorado surge al constatar que el alumnado sigue sus explicaciones. Le satisface también, la óptima y pronta resolución de los problemas cotidianos por parte de los implicados en el Programa.

La información cualitativa obtenida tras la realización de los grupos de discusión ha descubierto que los profesores implicados en el Programa se muestran altamente gratificados por la labor que desempeñan. Se hallan muy satisfechos al tener la 
oportunidad de trabajar con alumnos adultos mayores muy motivados y siempre con deseo de seguir aprendiendo.

Los profesores están satisfechos porque los alumnos les muestran su interés y afán de aprender, les gusta transmitir conocimientos a gente motivada. Se sienten gratificados cuando perciben que los mayores aprenden. En cuanto a la metodología y organización ponen en valor la flexibilidad en la enseñanza, la adecuación metodológica a las necesidades de las personas mayores y la oportunidad que se les ofrece para enriquecerse y adquirir nuevos conocimientos.

Con respecto a las relaciones interpersonales subrayan la interacción, relación y comunicación con los mayores; su ilusión, ganas y predisposición para aprender, así como el buen ambiente que se genera.

No obstante, los profesores sugieren algunos cambios que convendría introducir en orden a lograr un óptimo desarrollo del Programa, a saber: mejoras en la organización, metodología y recursos, en difusión, matriculación y coordinación.

En concreto en organización sugieren incrementar el horario y ampliar la duración del curso con la finalidad de afianzar los conocimientos.

Por último nos parecen importantes los aspectos asociados a la metodología, tales como la necesidad de favorecer más la implicación de los alumnos a través de dinámicas participativas, así como la importancia de crear redes de contacto para intercambio de experiencias en el centro y con otros centros.

\section{Discusión y conclusiones}

Los implicados en la UNED Senior, (alumnos, coordinadores y profesores) manifiestan una actitud muy positiva hacia este Programa formativo.

Los alumnos expresan su satisfacción al comprobar que la UNED Senior responde a lo que esperaban. Gozan de libertad para la elección de materias Les gustan las actividades programadas y su funcionamiento en los diferentes centros locales.

Por su parte, los coordinadores declaran que su mayor grado de satisfacción se halla al constatar que los profesores y alumnos están satisfechos con la organización y el funcionamiento de sus respectivos Centros Asociados.

Finalmente, los profesores expresan que su mayor satisfacción reside en evidenciar que el alumnado sigue sus explicaciones en las clases. Valoran también, que le resuelvan los problemas con prontitud.

Por otra parte, los resultados obtenidos han permitido identificar aquellos aspectos que más motivan a los alumnos para iniciar sus estudios en el Programa, tales como: aprender más, mantenerse activo, conocer a otras personas, sentirse útil...

Ello ratifica que los mayores demandan un papel activo en su proceso de enseñanza y aprendizaje, convirtiéndose en protagonistas de su propio aprendizaje para el logro y desarrollo de un envejecimiento activo. En este sentido Lirio y Morales (2011, p. 159) 
afirman que "los mayores aprenden a través de la construcción activa del conocimiento, es decir, participando".

Los profesores están muy satisfechos porque los alumnos asisten con regularidad a las clases. Muestran interés, motivación y son participativos. Un elemento de singular importancia es que las asignaturas las eligen voluntariamente. En este sentido, es conveniente seguir apostando por la flexibilidad del Programa y ofertar asignaturas que solicite el alumnado y despierten su interés. Desde esta óptica sería necesaria una mayor formación y especialización de los docentes para responder a las nuevas demandas.

A pesar de la importancia de los docentes en toda acción formativa todavía son escasos los programas dirigidos a la formación del profesorado para trabajar con adultos mayores.

Los profesores necesitan disponer de más recursos y una mejor formación que les permita adquirir las competencias y estrategias necesarias para dar una respuesta válida, realista y eficaz a los nuevos requerimientos de este colectivo.

El profesorado destaca que serían necesarias mejoras en el centro y en las aulas: adaptar el mobiliario, accesibilidad, equipos informáticos y audiovisuales. En este sentido es imprescindible, "facilitar el acceso de los colectivos más vulnerables, decisivo para su integración, participación e inserción social con los mismos derechos que el resto de los ciudadanos" (Pérez y Sarrate, 2011, p. 238).

Otro aspecto de interés es el clima existente en los diversos centros. Se valora positivamente la vinculación de profesores y alumnos con el centro. Aunque algunos profesores mantiene escasos contactos.

Los alumnos están satisfechos con el profesorado y alaban competencias, tales como: el conocimiento de la materia, las explicaciones pertinentes y, utilización de ejemplos claros; la aplicación de los contenidos a la vida cotidiana. Se preocupan de seguir aprendiendo y, por ello, se mantienen atentos a las explicaciones del profesor, dado que se hallan interesados por su óptima formación.

En esta investigación se ha tratado de ahondar en las necesidades de los participantes. Lo que permitirá adaptar y adecuar el Programa a sus necesidades, preferencias e intereses. De este modo, se podrán diseñar intervenciones prácticas que permitan superar las dificultades con las que se encuentren. En cada contexto, los responsables de la formación serán los encargados de adecuar el Programa siguiendo el principio de la flexibilidad.

Finalmente se señalan algunas sugerencias suscitadas en esta investigación con las que se pretende contribuir a la mejora de los PUM:

- Los PUM todavía son poco conocidos. Su oferta formativa no se ha difundido en las diferentes zonas del país. Un mejor conocimiento fomentaría nuevas oportunidades y apoyos para estimular a las personas mayores a participar. Esta situación se solventaría con la realización de campañas de publicidad y difusión a través de diferentes medios. 
- Es preciso contar con más materiales didácticos y medios tecnológicos (ordenadores, proyectores...) para su utilización. Gracias a las nuevas tecnologías las zonas rurales se pueden vincular a los programas universitarios de mayores, aprovechando las posibilidades de Internet. Favoreciendo de este modo no solo el aprendizaje compartido sino también las interrelaciones, la cooperación y participación.

- Los alumnos demandan mayor oferta formativa con el fin de tener más posibilidades para elegir las asignaturas que mejor respondan a sus necesidades e intereses.

- Al alumnado les gustaría que se prolongara la duración de las clases y las asignaturas se impartieran a lo largo del curso académico. Explicitan que son capaces de aprender pero precisan más tiempo para afianzar los conocimientos.

- Los participantes demandan una metodología activa y participativa. Acuden a las aulas con una trayectoria vital muy rica y con la sabiduría de la experiencia. Por ello, reclaman metodologías como: el trabajo en grupo, debates, lectura y análisis de la realidad, intercambio de experiencias, realización de actividades fuera del aula (culturales, excursiones, salidas...), aprovechando los recursos del entorno.

- Una mayor implicación de las distintas entidades públicas y privadas en la financiación de los programas contribuiría a su mejor reconocimiento.

- Desde el punto de vista de la organización y funcionamiento del Programa sería conveniente generar espacios de encuentro entre los implicados con el fin de abordar los diferentes aspectos relacionados con el diseño, organización y planificación teniendo en cuenta los intereses y necesidades de los participantes.

Las claves del éxito de los PUM - también de la UNED Senior - consisten en ofrecer una formación asequible y flexible, implicando a los participantes y a la sociedad en su conjunto. En este sentido se convierten en un modelo que propicia el aprendizaje a lo largo de la vida favoreciendo, de este modo, un envejecimiento activo, exitoso y saludable.

\section{Referencias bibliográficas}

AEPUM (2012). Nacimiento y desarrollo de los PUM. Asociación Estatal de programas universitarios para mayores. Boletín Monográfico de AEPUM. $\mathrm{n}^{\circ} .1,1$ 11. Recuperado

en http://www.aepumayores.org/sites/default/files/Boletin_Monografico_AEPUM.pdf (Consultado 3 de enero de 2013).

BERMEJO GARCÍA, L. (2010). Envejecimiento Activo y Actividades Socioeducativas con Personas Mayores. Guía de buenas prácticas. Sociedad Española de Geriatría y Gerontología. Serie Gerontología Social. México: Editorial Médica Panamericana. 
DELORS, J. et ál. (1996). Informe de la UNESCO. La educación encierra un tesoro. Madrid: Santillana.

GONZÁLEZ, I. (2012, octubre). Conferencia Ministerial sobre Envejecimiento. Cuadernos 60 y más. Año XVIII, no 314 octubre, pp. 29-36. Recuperado en http://www.imserso.es/InterPresent1/groups/imserso/documents/binario/s314cuader nos.pdf (Consultado 15 de enero de 2013).

HENSON, R. K. (2001). Understanding internal consistency reliability estimates: A conceptual primer on coefficient alpha. Measurement and Evaluation in Counseling and Development, 34 (3), 177-189.

JIMÉNEZ EGUIZÁBAL, A. y PALMERO CÁMARA, C. (coord.) (2012). Programas Universitarios para mayores en España. Memorialidades, $n^{\circ}$ 17, jan./jun. Pp. 510.

LEY ORGÁNICA 4/2007, de 12 de abril, por la que se modifica la Ley Orgánica 6/2001, de 21 de diciembre, de Universidades. Recuperado en http://www.boe.es/boe/dias/2007/04/13/pdfs/A16241-16260.pdf (Consultado 13 de mayo de 2013).

LIRIO CASTRO, J. y MORALES CALVO, S. (2011). "El reto de la formación el profesorado para una enseñanza de calidad en las universidades de mayores". Pedagogía Social. Revista Interuniversitaria, 19, pp. 155-166.

LOU. Ley orgánica 6/2001, de 21 de diciembre, de Universidades. Boletín Oficial del Estado. 24515. Recuperado en www.boe.es/boe/dias/2001/12/24/pdfs/A4940049425.pdf (Consultado 14 de febrero de 2013).

MINISTERIO DE TRABAJO Y ASUNTOS SOCIALES (2001). La OMS ante la II Asamblea Mundial del Envejecimiento. Salud y envejecimiento. Un documento para el debate. Boletín sobre el envejecimiento, $\mathrm{N}^{\mathrm{o}} 4-5$, Madrid.

MINISTERIO DE TRABAJO Y POLÍTICA SOCIAL (2011). Libro blanco. Envejecimiento Activo. Madrid: IMSERSO.

ONU. I Asamblea Mundial del Envejecimiento, Viena. Organizada por Naciones Unidas; 1982.

ORGANIZACIÓN MUNDIAL DE LA SALUD (2002). Envejecimiento activo: un marco político. Revista Española de Geriatría y Gerontología, Vol. 37, Suplemento 2, pp. 74-105.

PÉREZ SERRANO, G. (2011). El conocimiento científico y sus carcomas. Teoría de la Educación. Revista Interuniversitaria. Vol. 23, 2, diciembre. Págs. 19-43.

PÉREZ SERRANO, G. y SARRATE CAPDEVILA, M. L. (2011). Las Tic Promotoras de inclusión social. Revista Española de Pedagogía. Año LXIX. № 249, mayoagosto, 237-254.

UNESCO (1998). Declaración Mundial sobre la Educación Superior en el siglo XXI: visión y acción. Conferencia Mundial sobre la Educación Superior. Recuperado en 
http://www.unesco.org/education/educprog/wche/declaration_spa.htm (Consultado 3 de enero de 2013).

VILLA, J. M. (2007, junio). Jornada Científica: La Formación Permanente en la Estrategia Europea. Análisis y evaluación de las enseñanzas universitarias para mayores en España y Europa. Cuadernos 60 y más. Año XIII, nº 6 junio, pp. 31-33. Recuperado http://www.aepumayores.org/compartido/WEB/2006_07/dossier/revista60ymasJuni o.pdf (Consultado 3 de enero de 2013).

\section{Correspondencia con los autores}

Ana FERNÁNDEZ-GARCÍA

Universidad Nacional de Educación a Distancia

Facultad de Educación C/Juan del Rosal, 1428040 Madrid

e-mail: anafernandez@bec.uned.es

José Luis GARCÍA LLAMAS

Universidad Nacional de Educación a Distancia

Facultad de Educación C/Juan del Rosal, 1428040 Madrid

e-mail: jlgarcia@edu.uned.es

Gloria PÉREZ SERRANO

Universidad Nacional de Educación a Distancia

Facultad de Educación C/Juan del Rosal, 1428040 Madrid

e-mail: gloriaperez@edu.uned.es 